\title{
Differences in BRCA counseling and testing practices based on ordering provider type
}

\author{
Deborah Cragun, CGC, PhD ${ }^{1}$, Lucia Camperlengo, $\mathrm{MPH}^{1}$, Emily Robinson, $\mathrm{MPH}^{1}$, \\ Meghan Caldwell, MS, CGC ${ }^{1}$, Jongphil Kim, $\mathrm{PhD}^{1}$, Catherine Phelan, MD, PhD, \\ Alvaro N. Monteiro, PhD ${ }^{1}$, Susan T. Vadaparampil, PhD, $\mathrm{MPH}^{1}$, \\ Thomas A. Sellers, PhD, MPH ${ }^{1}$ and Tuya Pal, MD
}

Purpose: The purpose of this study was to assess potential differences in genetic counseling services delivered by board-certified genetic health-care providers versus non-genetic health-care providers. We evaluated (i) patient recall and content of pretest genetic counseling for hereditary breast and ovarian cancer and (ii) whether full BRCA1 and 2 gene sequencing was performed when less expensive single-site or Ashkenazi Jewish founder mutation testing may have been sufficient.

Methods: Participants completed a questionnaire and provided $B R C A$ test reports that included testing provider and type of test. Chi-square tests and logistic regression were used for analysis.

Results: Of 473 participants, $>90 \%$ were white, female, and $B R C A$ mutation carriers. Of the 276 (58\%) with genetic health-care provider involvement, $97 \%$ recalled a pretest discussion as compared with $59 \%$ of those without genetic health-care provider involvement
$(P<0.001)$. Among the subgroup who recalled a pretest discussion $(n=385)$, those with genetic health-care provider involvement indicated higher adherence to eight recognized genetic counseling elements, four of which were statistically significant. Furthermore, involvement of a genetic health-care provider halved the likelihood that comprehensive $B R C A$ testing was ordered among the 266 for whom single-site or multisite-3 testing may have been sufficient $(P=0.02)$.

Conclusion: Our results suggest that genetic health-care provider involvement is associated with adherence to nationally recommended genetic counseling practices and could potentially reduce costs of BRCA genetic testing.

Genet Med advance online publication 12 June 2014

Key Words: genetic counseling; genetic testing; hereditary breast and ovarian cancer syndrome; informed consent

\section{INTRODUCTION}

Approximately $10 \%$ of breast cancers are due to highly penetrant inherited gene mutations, ${ }^{1-3}$ the majority of which occur in the BRCA1 and BRCA2 (BRCA) genes. ${ }^{4,5}$ Clinical testing for $B R C A$ enables the identification of individuals at greatly elevated lifetime risk for hereditary breast and ovarian cancer, in the range of $60-70 \%$ and $40 \%$, respectively ${ }^{6-8}$ In $B R C A$ carriers, these high cancer risks can be reduced through proven cancer prevention and early detection options. ${ }^{9}$

Since the discovery of the BRCA genes more than a decade ago, an increasing number of patients have been tested for hereditary cancer in the community setting without involvement of a genetic health-care provider (GHP) (i.e., boardcertified genetic counselor or medical geneticist). ${ }^{10,11}$ Surveys of US providers estimate that $\sim 30 \%$ of primary-care physicians have ordered a genetic test for hereditary breast cancer. ${ }^{12,13}$ Regardless of who facilitates testing, several organizations have outlined elements that should be included as part of a pretest discussion to ensure quality of cancer genetic counseling (GC) services. ${ }^{14-16}$ Table 1 lists several common elements of pretest GC and informed consent outlined by the American Society of Clinical Oncology ${ }^{15}$ and the National Society of Genetic Counselors. ${ }^{14}$ Prior studies comparing delivery of GC services suggest that GHPs may be more likely to spend a longer amount of time in pretest counseling; ${ }^{11,17}$ however, content of sessions and patient recall of recommended elements remains uncertain.

Provider surveys based on hypothetical scenarios have suggested deficiencies in knowledge of non-GHPs that may lead to inappropriate or more costly testing. ${ }^{18,19}$ However, no patientlevel data to evaluate testing based on GHP involvement have been reported. When ordering clinical BRCA testing, some options include (i) comprehensive sequencing and rearrangement testing of BRCA1 and BRCA2, (ii) single-site testing for a known familial $B R C A$ gene mutation, or (iii) testing for three $B R C A$ mutations that comprise the vast majority of mutations within the Ashkenazi Jewish (AJ) population (commonly referred to as multisite- 3 by one of the testing laboratories). ${ }^{20}$ Although prices have fluctuated, the two latter options cost about 10-fold less than comprehensive gene sequencing and rearrangement testing (i.e., under $\$ 400$ vs. $\$ 3,000-\$ 4,000$ ). ${ }^{21}$

The current study sought to compare genetic services based on involvement of GHPs using patient-reported data. 
Table 1 Recommended elements of pretest counseling and related study questions

ASCO $^{\mathrm{a}}$

Pedigree information should be used

Collect 3- to 4-generation family medical history

Recognize indications for genetic cancer predisposition testing

Possibility that test will not be informative

Risk of passing mutation on to children and importance of sharing result

Options/limitations of medical surveillance and prevention strategies

NSGC

Identify potential hereditary cancer syndromes that should be considered

Possibility of variant of uncertain significance

Mode of inheritance associated with the gene and importance of sharing result

Options for cancer risk reduction and surveillance based on the patient's risk
Study question

Did you have a 3-generation family tree drawn to obtain a medical history of relatives from both your mother's and father's sides of your family?

Did your health-care provider discuss the possibility of other hereditary cancer syndromes?

Did your health-care provider discuss the possibility that the test will not be informative (i.e., variant of uncertain significance result)?

Did your health-care provider discuss what results might mean for your other family members?

Did your health-care provider discuss how results of genetic testing might change your future medical care?
Risks of insurance or employer discrimination

Extent to which laws are protective for health life, and disability insurance

a) Did your health-care provider discuss laws that protect against genetic discrimination by health insurers and employers?

b) Did your health-care provider discuss how genetic testing may impact life insurance and disability insurance?

Not mentioned Provide patient with tools to inform and educate family members (i.e., family contact letter) ${ }^{c}$
Did you receive a letter that summarized the information discussed during your counseling session?

The elements in this table do not comprise a comprehensive list.

ASCO, American Society of Clinical Oncology; NSGC, National Society of Genetic Counselors.

aData taken from ref. 15. 'Data taken from ref. 14. The patient letter has long been recognized as a valuable tool of genetic counseling that is often shared with other family members. ${ }^{31}$ Furthermore, a patient summary letter is included in the minimum guidelines for the delivery of clinical genetics services that were generated by the Great Lakes Regional Genetics Group. 28

We hypothesized that GHP involvement would (i) increase the likelihood that patients recalled having a pretest discussion and specific elements of GC and (ii) decrease the likelihood that patients would have comprehensive BRCA testing when less expensive testing (i.e., of single-site or multisite-3 AJ founder mutations) may be sufficient.

\section{Study population}

\section{MATERIALS AND METHODS}

Following approval by the University of South Florida's institutional review board in 2010, individuals with a personal and/or family history of cancer who were living in the United States were offered enrollment in the Inherited Cancer Registry. Participants were recruited through various clinical centers, directly online through the registry website (http://www. inheritedcancer.net), or through local and national outreach activities. $^{17}$

For the current study, eligibility criteria included Inherited Cancer Registry participants with prior BRCA testing who enrolled between 2010 and 2013, who completed a follow-up questionnaire, and for whom a copy of their $B R C A$ test report (471 cases) or medical record documentation of both test result and ordering provider (two cases) were available. Participants received genetic testing at the discretion of their treating health-care providers through a commercial US laboratory prior to enrollment. Genetic testing criteria were determined by their treating health-care provider and were not dictated by the study protocol.

\section{Procedures and measures}

A cross-sectional survey was conducted to collect demographic, clinical, and family history information. Recall of pretest GC was measured by asking, "Before you had your blood drawn for the genetic test, did you discuss the risks and benefits of testing (referred to as "Genetic Counseling")." Those who responded yes were then asked a multipart question about whether they recalled eight nationally recommended GC elements listed in Table 1.

Following signed medical release, study participants provided a copy of their BRCA test results or had test results or other medical records collected by study staff. Public websites were searched to determine the specialty of the ordering providers listed on the test reports and to identify whether they routinely work with a board-eligible/boardcertified genetic counselor. GHPs were defined as boardcertified genetic counselors or MD clinical geneticists. In cases in which more than one provider was listed on the test result $(n=268)$, provider type was categorized according to the nonphysician provider (e.g., nurse, nurse practitioner, or genetic counselor).

The type of $B R C A$ test ordered (i.e., comprehensive versus single-site or AJ founder mutations) was abstracted from the test report. Two patient-reported survey questions were used to identify cases for which single-site or AJ founder mutation testing may have been appropriate. These included (i) "Were you the first person in your family to be tested for a BRCA mutation?" and (ii) "Do you have Ashkenazi Jewish ancestry?" 


\section{Statistical analysis}

Participant characteristics were summarized using descriptive statistics, including medians and ranges for continuous variables and frequencies and proportions for categorical variables. Pearson $\chi^{2}$ tests and Mann-Whitney $U$-tests were used to compare characteristics of participants according to GHP involvement. Simple logistic regression modeling was used to test hypotheses. Multiple logistic regression modeling using backward elimination was performed to adjust for the effects

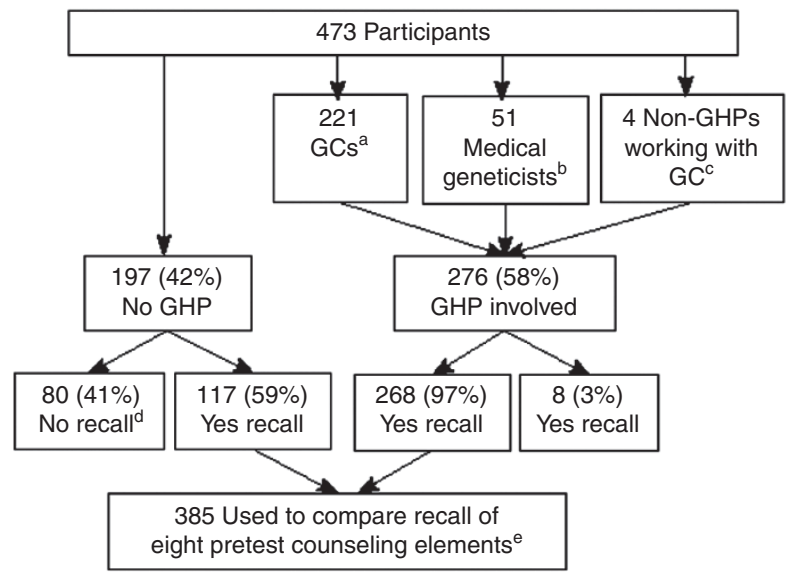

GC, master's degree-trained genetic counselor; GHP, genetic health-care provider (i.e., board certified GC or medical geneticist).

${ }^{a} \mathrm{GC}$ listed on test result with another health-care provider. ${ }^{\mathrm{b}}$ Medical geneticist listed on test result without a GC listed. ' Non-genetic health-care providers listed on test result working routinely with GC. ${ }^{d}$ Includes 5 who were unsure whether they had a pretest discussion. ${ }^{\mathrm{e}}$ Table 3 shows results comparing recall of pretest counseling elements.

Figure 1 Type of provider listed on BRCA test result and participants' recall of pretest discussion according to involvement of a genetic health-care provider. of potential confounders (i.e., AJ ancestry, first in family tested, years since testing, and $B R C A$ carrier status); the $P$ value for remaining in the model was set at 0.15 . All tests were two sided, and $P$ values were considered significant if $<0.05$. Analyses were performed using SPSS version 21 (SPSS, Chicago, IL).

\section{Participants}

\section{RESULTS}

A total of 473 individuals from 43 states met eligibility criteria for the current study. As depicted in Figure 1, a master's degree-trained genetic counselor or board-certified medical geneticist was listed on 272 test results. The ordering providers for four additional participants practice at National Cancer Institute-designated comprehensive cancer centers and work routinely with genetic counselors. Thus, 276 participants (58\%) had evidence of GHP involvement. Among the 197 remaining participants, provider specialty included obstetrician/gynecologist $(n=37)$, surgeon/surgical oncologist $(n=24)$, oncologist $(n=47)$, advanced practice nurse/nurse practitioner $(n=$ $36)$, nurse $(n=21)$, and other health-care provider $(n=32)$. Table 2 shows participant demographics for the entire sample and according to involvement of a GHP. More than $90 \%$ of participants were white, non-Hispanic, female, and BRCA carriers.

\section{Recall of pretest discussion and components of genetic risk assessment and counseling}

Among those with GHP involvement, 97\% recalled having a pretest discussion as compared with 59\% without GHP involvement $(P<0.001)$. Covariates (i.e., years since testing, AJ ancestry, first in family tested, $B R C A$ carrier status, and personal history of cancer) did not remain in the final model after

Table 2 Participant demographics and comparisons according to involvement of a genetic health-care provider (GHP)

\begin{tabular}{|c|c|c|c|c|}
\hline \multirow[b]{2}{*}{ Characteristic } & \multirow[b]{2}{*}{ All participants $(N=473)$} & \multicolumn{2}{|c|}{ GHP involved } & \multirow[b]{2}{*}{$P$ value } \\
\hline & & No $(n=197)$ & Yes $(n=276)$ & \\
\hline Median current age (Q1, Q3) & $52(44,59)$ & $52(44,59)$ & $51(43,59)$ & 0.58 \\
\hline Gender female (\%) & $461(97.5)$ & $193(98.0)$ & $268(97.1)$ & $0.77^{a}$ \\
\hline Married (\%) & $319(67.4)$ & $139(70.6)$ & $180(65.2)$ & 0.22 \\
\hline White, non-Hispanic (\%) & $439(92.8)$ & $184(93.4)$ & $255(92.4)$ & 0.67 \\
\hline Ashkenazi Jewish (\%) & $120(25.4)$ & $41(20.8)$ & 79 (28.6) & 0.05 \\
\hline$B R C A$ carrier $(\%)$ & $443(93.7)$ & $191(97.0)$ & $252(91.3)$ & 0.01 \\
\hline First tested in family (\%) & $239(50.5)$ & $106(53.8)$ & $133(48.2)$ & 0.23 \\
\hline Median years since testing (Q1, Q3) & $5(3,7)$ & $4(3,6)$ & $5(3,8)$ & 0.01 \\
\hline Median age at testing (Q1, Q3) & $46(38,54)$ & $46(39,54)$ & $45(38,53)$ & 0.14 \\
\hline Personal history of breast cancer (\%) & $174(36.8)$ & $75(38.1)$ & $99(35.9)$ & 0.62 \\
\hline Personal history of any cancer (\%) & $255(53.9)$ & $104(52.8)$ & $151(54.7)$ & 0.53 \\
\hline \multicolumn{5}{|l|}{ Education (\%) } \\
\hline Postgraduate degree & $165(34.9)$ & $62(31.5)$ & $103(37.3)$ & 0.58 \\
\hline Graduated college & $159(33.6)$ & $71(36.0)$ & $88(31.9)$ & - \\
\hline Some college & 75 (15.9) & $35(17.8)$ & $40(14.5)$ & - \\
\hline No college & $33(7.0)$ & $12(6.5)$ & $21(8.3)$ & - \\
\hline Unknown & $41(8.7)$ & $17(8.6)$ & $24(8.7)$ & - \\
\hline
\end{tabular}

Q1, lower quartile; Q3, upper quartile.

aYates' correction was employed due to low expected frequencies in $>20 \%$ of cells. 
Table 3 Patient recall of various elements of genetic counseling according to involvement of a genetic health-care provider (GHP)

\begin{tabular}{|c|c|c|c|}
\hline \multirow[b]{2}{*}{$\begin{array}{l}\text { Element of genetic } \\
\text { counseling }\end{array}$} & \multicolumn{2}{|c|}{ GHP involved } & \multirow[b]{2}{*}{$P$ value } \\
\hline & $\begin{array}{c}\text { No } \\
(n=117)^{\mathrm{a}}\end{array}$ & $\begin{array}{c}\text { Yes } \\
(n=268)^{\mathrm{a}}\end{array}$ & \\
\hline Pedigree completed & $82(82.8)$ & $261(91.3)$ & 0.01 \\
\hline \multicolumn{4}{|l|}{ Discussion of: } \\
\hline $\begin{array}{l}\text { Variants of uncertain } \\
\text { significance }\end{array}$ & $72(72.7)$ & $229(80.1)$ & 0.23 \\
\hline $\begin{array}{l}\text { Laws protecting against } \\
\text { health insurance } \\
\text { discrimination }\end{array}$ & $73(73.7)$ & $237(82.9)$ & 0.01 \\
\hline $\begin{array}{l}\text { Issues related to disability } \\
\text { insurance }\end{array}$ & $57(57.6)$ & $203(71.0)$ & 0.01 \\
\hline Other hereditary cancers & $63(63.9)$ & $199(69.6)$ & 0.12 \\
\hline $\begin{array}{l}\text { Management } \\
\text { recommendations }\end{array}$ & $82(82.8)$ & $244(85.3)$ & 0.35 \\
\hline Family implications & 91 (91.9) & $273(95.5)$ & 0.21 \\
\hline Summary letter received & $58(58.6)$ & $226(79.0)$ & $<0.001$ \\
\hline
\end{tabular}

anly the 385 participants who remembered having a pretest discussion were asked whether they recalled each of the elements of pretest genetic counseling. ${ }^{b}$ Results of simple logistic regression (shown here) were more conservative than results of multiple logistic regression.

backward elimination; thus, these covariates did not appear to influence recall of pretest discussion nor did they attenuate the relationship between recall and GHP involvement.

Among the subset of participants who recalled a pretest discussion $(n=385)$, those with GHP involvement were more likely to also recall specific GC elements, and these differences were statistically significant for four of the eight elements, including (i) having a pedigree drawn; (ii) discussing laws protecting against genetic discrimination by health insurers; (iii) discussing issues related to life or disability insurance; and (iv) being sent a summary letter (Table 3). Adjustment for years since testing, AJ ancestry, first in family tested, BRCA carrier status, and personal history of cancer did not attenuate these differences.

\section{Type of test ordered}

A subset of 266 participants were of AJ ancestry $(n=147)$, had a prior family member tested $(n=61)$, or both $(n=58)$. Among this subset, 63.2 and $36.8 \%$ were tested with and without GHP involvement, respectively. Comprehensive testing was performed in $9.5 \%$ of the GHP group as compared with $19.4 \%$ of the non-GHP group $(P=0.02)$. BRCA carrier status, personal history of cancer, and years since testing did not account for the difference.

\section{DISCUSSION}

The current study suggests that a pretest discussion occurs significantly more often when a GHP is involved in BRCA testing, with a higher likelihood that specific nationally recommended GC elements are discussed. ${ }^{14,15}$ Furthermore, among those for whom less expensive testing may have been sufficient (i.e., those with single-site or multisite- $3 \mathrm{AJ}$ founder mutations), the more costly full gene sequencing was approximately twice as likely to be ordered when testing was performed without GHP involvement.

Several professional organizations have recommended pretest GC prior to ordering $B R C A$ testing and outlined specific elements to be discussed. ${ }^{14-16,22}$ Despite these national guidelines, our findings indicate that many patients seen by non-GHPs do not recall receiving the recommended pretest information, which is necessary to make an informed decision about testing. In fact, more than $40 \%$ of patients tested without GHP involvement failed to recall any pretest discussion, as compared with only $3 \%$ of patients tested with GHP involvement. Although we were unable to identify prior patient studies comparing recall of a pretest discussion by provider type, a study of 646 patients who received $B R C A$ testing in a nonacademic setting reported that $82 \%$ recalled discussing and reviewing the consent form with some type of health-care provider (including 319 genetic counselors, 162 physicians, and 41 nurses). ${ }^{23}$ This prior study did not specify proportions who recalled the informed consent discussion by provider type, nor did it report on specific elements included as part of the discussion.

Although survey data among patients tested by non-GHPs are limited, a few provider surveys assessed $B R C A$ testing services across diverse settings. One study of 611 physicians at nonacademic centers surveyed in 2004-2005 found that almost 70\% discussed six counseling items prior to testing. ${ }^{11}$ Discussion of the six elements was associated with physicians who worked with genetic counselors, nurse geneticists, or other health-care professionals (versus counseling by themselves), as well as with those who spend more than 60 minutes counseling. These results may be contrasted with findings from our 2012 survey of 81 nonGHPs, in which the majority of providers reported GC sessions of 30 minutes or less, with many reporting limited or no discussion of recommended pretest elements. ${ }^{24}$ Thus, our recent providerlevel data are consistent with findings from the current study, in which more than $40 \%$ of participants tested by non-GHPs did not recall having had pretest GC. However, our data also suggest that many non-GHPs are performing pretest counseling that includes discussion of many nationally recommended GC elements. In fact, among the subset of study participants who recalled having a pretest discussion, the majority indicated that most of the GC elements were discussed; this was true regardless of whether a GHP was involved. Nevertheless, our data indicate that recall of each element was consistently higher with GHP involvement, although differences were only statistically significant for implications of testing on life and disability insurance-related issues and laws protecting against discrimination by health insurers and employers. These findings are particularly important when considering testing for inherited cancer in individuals without a prior cancer diagnosis, because they may have concerns about discrimination, and identification of a mutation may be considered a preexisting condition when applying for life or disability insurance but not for health insurance.

Another important finding of the current study is that recall of having a three-generation pedigree completed was significantly 
more common with GHP involvement. This finding is consistent with prior studies that found a minority of non-GHPs consistently draw a pedigree or adequately document family history. ${ }^{25-27}$ Potential implications of incomplete family history include inaccurate or incomplete risk assessment, which may have implications for patient care or may result in inappropriate testing or failure to identify at-risk relatives who may also need testing. Moreover, our results indicated that patients received a summary letter more often when a GHP was involved, which is standard clinical practice in genetics. ${ }^{28}$ Prior studies have indicated that copying of correspondence to patients is associated with a range of benefits including greater patient understanding, greater patient involvement, reduction in anxiety, increased satisfaction, and more accurate risk perception. ${ }^{29,30}$ Furthermore, a letter provides something that patients can share with relatives. ${ }^{31}$

Our findings suggest that there may be potential cost-of-care implications associated with GHP involvement, because less costly, single-site, or AJ mutation testing was more likely to be ordered in appropriate cases when a GHP was involved. Our results concur with trends in provider surveys of hypothetical scenarios, which have found that non-GHPs are more likely to indicate that they would order the more expensive comprehensive testing in patient scenarios in which less expensive testing would be appropriate. ${ }^{18,19}$ However, aside from these hypothetical scenarios, there are very limited published data to evaluate actual genetic testing practices by various health-care providers. One of the few publications available describes the experience of a US-based clinical laboratory and concludes that inappropriate ordering of genetic tests is common, occurring in $\sim 26 \%$ of all complex genetic tests assessing germ-line mutations. ${ }^{32}$ Furthermore, having laboratory genetic counselors review tests ordered to ensure appropriateness of testing led to substantial cost savings. ${ }^{32}$ BRCA tests were not included in that report because the study was conducted when all clinical BRCA testing in the Unites States occurred at the single clinical laboratory that held the BRCA gene patents (until 2013). Unfortunately, overall health-care expenditure on $B R C A$ testing is difficult to estimate, given the lack of published data regarding the number and type of BRCA tests completed each year. However, a large national insurer found that spending per member on molecular and genetic tests increased by about $14 \%$ a year on average between 2008 and 2010, and the insurer expects these costs to continue increasing. ${ }^{33}$

Despite the paucity of published peer-reviewed studies comparing testing costs or quality of $B R C A$ pretest counseling according to GHP involvement, Cigna became the first national insurer to implement a policy whereby GC by a certified genetics professional (which Cigna defined as a board-certified genetic counselor or medical geneticist) is required prior to $B R C A$ testing and upon which coverage decisions for the test are based. ${ }^{34,35}$ This change in policy was reportedly implemented to enhance quality of care while recognizing that $20 \%$ of tests are ordered inappropriately. ${ }^{34,35}$ Cigna's policy was welcomed by the National Society of Genetic Counselors, which stated that it will result in more individuals receiving appropriate genetic testing and making informed decisions about their health. ${ }^{36}$ By contrast, the response from the American Society of Clinical Oncology stated that this policy poses risks to patients by serving as a barrier to the appropriate use of genetic testing services. $^{37}$

This recent policy-level development highlights the need to generate data to guide cost-effective delivery of genetic testing services while optimizing health benefits for patients. Our study adds to limited evidence regarding how and by whom the delivery of hereditary cancer genetic services may maximize the likelihood of high-quality, cost-effective GC services. Although our data suggest that several providers who are not certified in genetics appear to provide adequate pretest GC, the issue remains that their credentials do not easily distinguish them from other providers who fail to provide adequate pretest GC.

Thus, our data highlight complexities that arise when making policy-level decisions and support the need to consider new service-delivery models. For example, innovative approaches to delivering quality genetic services to an increasing number of patients in community settings have been demonstrated through establishing academic-community partnerships that focus on collaboration with non-GHPs in which GHPs serve as a hub of information about the GC and testing processes. ${ }^{38}$

Our study has several strengths, including our relatively large sample of BRCA carriers and recruitment of participants who had genetic testing through providers of various specialties across the United States. Furthermore, collection of the genetic test report enabled us to verify whether a GHP was involved. In addition, post hoc sensitivity analyses determined our findings to be robust even when the four cases for whom GHP involvement was inferred were either removed or included in the non-GHP group and even when the two participants for whom results were obtained through medical record review were removed. Despite these strengths, there remain limitations resulting from the observational design of the study with collection of retrospective patient-reported data that may be subject to recall bias. Although we have no reason to believe there would be differences in recall bias based on involvement of a GHP, time from testing to consenting for our study was actually longer for the group tested by GHPs. After controlling for time since testing and several other potential confounders, differences in recall between the GHP and non-GHP groups remained similar or increased, indicating that our results may actually underestimate the true differences between those tested with and without GHP involvement. Another limitation is the lack of diversity in our sample, given that our participants were primarily highly educated and Caucasian; this is a limitation observed in most prior studies of individuals tested for $B R C A$ mutations, given the disparity in access to testing in minorities. ${ }^{20,39,40}$

The research gap addressed through our study is highlighted by the recent evidence-based BRCA testing guidelines issued by the US Preventive Services Task Force indicating that there is a need to generate data about the provision of genetic testing 
services by various providers in real-world settings. ${ }^{22}$ However, it would be important to replicate our findings in an ethnically and racially diverse population in order to develop ways to optimize delivery of GC and testing services across diverse populations. Furthermore, longitudinal follow-up in our cohort is important to determine whether and how differences in pretest discussions influence patient outcomes. In addition, larger studies will be necessary in order to compare service delivery among various non-GHP providers such as oncologists, surgeons, and nurses.

Ultimately, our study is among the first focused efforts to compare the delivery of hereditary cancer pretest GC services from the perspective of participants tested by various providers in diverse settings. Our results clearly suggest differences in delivery of services based on involvement of a GHP, including a higher likelihood of (i) pretest GC and discussion of recommended pretest GC elements and (ii) less expensive single-site and $\mathrm{AJ}$ testing when appropriate. These findings are important because policy-level decisions are made in the changing healthcare environment within the Unites States as the focus shifts to improving quality and cost effectiveness of health-care delivery.

\section{ACKNOWLEDGMENTS}

This work was supported by grants through Florida Biomedical (IBG09-34198 and 4BB15). Support for D.C.'s time was provided by a NCI R25T training grant awarded to Moffitt Cancer Center (5R25CA147832-04).

\section{DISCLOSURE}

The authors declare no conflict of interest.

\section{REFERENCES}

1. Filippini SE, Vega A. Breast cancer genes: beyond BRCA1 and BRCA2. Front Biosci (Landmark Ed) 2013;18:1358-1372.

2. Beggs $A D$, Hodgson SV. Genomics and breast cancer: the different levels of inherited susceptibility. Eur J Hum Genet 2009;17:855-856.

3. Stratton MR, Rahman N. The emerging landscape of breast cancer susceptibility. Nat Genet 2008;40:17-22.

4. Miki Y, Swensen J, Shattuck-Eidens D, et al. A strong candidate for the breast and ovarian cancer susceptibility gene BRCA1. Science 1994;266:66-71.

5. Wooster R, Bignell G, Lancaster J, et al. Identification of the breast cancer susceptibility gene BRCA2. Nature 1995;378:789-792.

6. Antoniou A, Pharoah PD, Narod S, et al. Average risks of breast and ovarian cancer associated with BRCA1 or BRCA2 mutations detected in case Series unselected for family history: a combined analysis of 22 studies. Am J Hum Genet 2003;72:1117-1130.

7. Chen S, Parmigiani G. Meta-analysis of BRCA1 and BRCA2 penetrance. J Clin Oncol 2007;25:1329-1333.

8. Pal T, Permuth-Wey J, Betts JA, et al. BRCA1 and BRCA2 mutations account for a large proportion of ovarian carcinoma cases. Cancer 2005;104:2807-2816.

9. Pal T, Vadaparampil ST. Genetic risk assessments in individuals at high risk for inherited breast cancer in the breast oncology care setting. Cancer Control 2012;19:255-266.

10. Cohen SA, Gustafson SL, Marvin ML, et al. Report from the National Society of Genetic Counselors service delivery model task force: a proposal to define models, components, and modes of referral. J Genet Couns 2012;21:645-651.

11. Keating NL, Stoeckert KA, Regan MM, DiGianni L, Garber JE. Physicians' experiences with BRCA1/2 testing in community settings. J Clin Oncol 2008:26:5789-5796.

12. Bellcross CA, Kolor K, Goddard KA, Coates RJ, Reyes M, Khoury MJ. Awareness and utilization of BRCA1/2 testing among U.S. primary care physicians. Am J Prev Med 2011;40:61-66.
13. Shields $A E$, Burke W, Levy DE. Differential use of available genetic tests among primary care physicians in the United States: results of a national survey. Genet Med 2008; 10:404-414.

14. Riley BD, Culver JO, Skrzynia C, et al. Essential elements of genetic cancer risk assessment, counseling, and testing: updated recommendations of the National Society of Genetic Counselors. J Genet Couns 2012;21: 151-161.

15. Robson ME, Storm CD, Weitzel J, Wollins DS, Offit K; American Society of Clinical Oncology. American Society of Clinical Oncology policy statement update: genetic and genomic testing for cancer susceptibility. J Clin Oncol 2010;28:893-901.

16. National Accreditation Program for Breast Centers. Genetic Evaluation and Management (page 48) 2013. http://napbc-breast.org/ standards/2013standardsmanual.pdf. Accessed 16 April 2014.

17. Pal T, Lee JH, Besharat $A$, et al. Modes of delivery of genetic testing services and the uptake of cancer risk management strategies in BRCA1 and BRCA2 carriers. Clin Genet 2014:85:49-53.

18. Pal T, Cragun D, Lewis $C$, et al. A statewide survey of practitioners to assess knowledge and clinical practices regarding hereditary breast and ovarian cancer. Genet Test Mol Biomarkers 2013;17:367-375.

19. Plon SE, Cooper HP, Parks B, et al. Genetic testing and cancer risk management recommendations by physicians for at-risk relatives. Genet Med 2011;13: 148-154.

20. Hall MJ, Reid JE, Burbidge LA, et al. BRCA1 and BRCA2 mutations in women of different ethnicities undergoing testing for hereditary breast-ovarian cancer. Cancer 2009;115:2222-2233.

21. Genetic Testing Facilities and Cost. http://www. breastcancer.org/symptoms/ testing/genetic/facility_cost. Accessed 15 April 2014.

22. Moyer VA; U.S. Preventive Services Task Force. Risk assessment, genetic counseling, and genetic testing for BRCA-related cancer in women: U.S. Preventive Services Task Force Recommendation Statement. Ann Intern Med 2014; 160:271-281

23. Chen WY, Garber JE, Higham $S$, et al. BRCA1/2 genetic testing in the community setting. J Clin Onco/ 2002;20:4485-4492.

24. Vadaparampil ST, Scherr CL, Cragun D, Malo TL, Pal T. Pre-test genetic counseling services for hereditary breast and ovarian cancer delivered by nongenetics professionals in the state of Florida. Clin Genet 2014; e-pub ahead of print 16 April 2014.

25. Wideroff L, Vadaparampil ST, Breen N, Croyle RT, Freedman AN. Awareness of genetic testing for increased cancer risk in the year 2000 National Health Interview Survey. Community Genet 2003;6:147-156.

26. Vig HS, Armstrong J, Egleston BL, et al. Cancer genetic risk assessment and referral patterns in primary care. Genet Test Mol Biomarkers 2009;13: 735-741.

27. Wood ME, Kadlubek P, Pham TH, et al. Quality of cancer family history and referral for genetic counseling and testing among oncology practices: a pilot test of quality measures as part of the American Society of Clinical Oncology Quality Oncology Practice Initiative. J Clin Oncol 2014;32:824-829.

28. Weaver $\mathrm{DD}$, Baker $\mathrm{D}$, Theobald $\mathrm{M}$, et al. Minimum guidelines for the delivery of clinical genetics services. The Evaluation of Clinical Services Subcommittee, Great Lakes Regional Genetics Group. Am J Hum Genet 1993;53:287-289.

29. Baxter S, Farrell K, Brown C, Clarke J, Davies H. Where have all the copy letters gone? A review of current practice in professional-patient correspondence. Patient Educ Couns 2008;71:259-264.

30. Lobb EA, Butow PN, Barratt $A$, et al. Communication and information-giving in high-risk breast cancer consultations: influence on patient outcomes. $\mathrm{Br}$ J Cancer 2004;90:321-327.

31. Hallowell N, Murton F. The value of written summaries of genetic consultations. Patient Educ Couns 1998;35:27-34

32. Miller CE, Krautscheid P, Baldwin EE, et al. Genetic counselor review of genetic test orders in a reference laboratory reduces unnecessary testing. Am J Med Genet A 2014;164A:1094-1101.

33. UnitedHealth Group. Personalized medicine: trends and prospects for the new science of genetic testing and molecular diagnostics. 2012. http://www. unitedhealthgroup.com/Newsroom/Articles/News/UnitedHealth\%20Group/20 12/0312WhitePaper7PersonalizedMedicine.aspx. Accessed 9 May 2014

34. Cigna. Genetic Testing and Counseling Program. http://www.cigna.com/ healthcare-professionals/resources-for-health-care-professionals/genetictesting-and-counseling-program. Accessed 15 April 2014

35. Lee J. Cigna to require counseling prior to some genetic tests. 2013. http:// www.modernhealthcare.com/article/20130725/NEWS/307259958/cigna-torequire-counseling-prior-to-some-genetic-tests. Accessed 15 April 2014. 
36. Genetic Counseling Program Gives Cigna Customers Increased Access to Genetic Counselors. NSGC Executive Office 2013. http://www.nsgc.org/p/bl/ et/blogid=45\&blogaid=20. Accessed 15 April 2014

37. American Society of Clinical Oncology. New Cigna Policy on Cancer Genetic Testing Poses Risks to High Quality Cancer Care. 2013. http://www.asco.org/ advocacy/new-cigna-policy-cancer-genetic-testing-poses-risks-high-qualitycancer-care. Accessed 15 April 2014
38. Cohen SA, Mcllvried D, Schnieders J. A collaborative approach to genetic testing: a community hospital's experience. J Genet Couns 2009;18:530-533.

39. Armstrong K, Micco E, Carney A, Stopfer J, Putt M. Racial differences in the use of BRCA1/2 testing among women with a family history of breast or ovarian cancer. JAMA 2005:293:1729-1736.

40. Forman $A D$, Hall MJ. Influence of race/ethnicity on genetic counseling and testing for hereditary breast and ovarian cancer. Breast J 2009;15(suppl 1):S56-S62. 